\title{
Manejo de resíduos sólidos na região sul do Brasil'
}

\author{
Solid waste management in southern Brazil
}

\author{
Rosane Maria Kirchner ${ }^{2}$, Jaqueline Silinske ${ }^{3}$, Joana Kirchner Benetti', Magda Antunes de Chaves ${ }^{5}$, \\ Mônica Elisa Scherer ${ }^{6}$, Ana Paula Fleig Saidelles ${ }^{7}$, Liliana Essi ${ }^{8}$. \\ ' Projeto de extensão com apoio PIBIC \\ 2 Doutora em Engenharia Elétrica- Métodos de Apoio à Decisão, Departamento de Zootecnia e \\ Ciências Biológicas da Universidade Federal de Santa Maria (UFSM). \\ ${ }^{3}$ Acadêmica do Curso de Administração \\ ${ }^{4}$ Engenheira Civil, Professora da UNIJUI \\ ${ }^{5}$ Acadêmica do Curso de Ciências Biológicas, UFSM: \\ ${ }^{6}$ Acadêmica do Curso de Administração, UFSM \\ ${ }^{7}$ Docente de Química, Doutora em Química, Universidade Federal do Pampa \\ ${ }^{8}$ Docente de Biologia, Doutora em Botânica, UFSM
}

\section{Resumo}

A grande quantidade de resíduos sólidos gerados pela população ocasiona problemas sociais, ambientais e de saúde, por isso, é importante que nos municípios do Brasil existam programas de manejo de resíduos sólidos com o intuito de diminuir os reflexos da má gestão desses materiais. Diante disso, o objetivo deste trabalho foi caracterizar o manejo de resíduos sólidos na região Sul do Brasil, sendo que para análise de dados foi utilizada estatística descritiva. Os dados são provenientes do IBGE referentes à Pesquisa Nacional de Saneamento Básico de 2008. Com este estudo, foi possível perceber que todos os municípios da região Sul do Brasil possuem manejo de resíduos sólidos, porém não existe coleta seletiva em 52,4\% dos municípios do Paraná, 66,6\% dos municípios de Santa Catarina e 66,5\% dos municípios do Rio Grande do Sul. No Brasil, a maior quantidade diária de resíduos sólidos é destinada a aterros sanitários, correspondendo a 64,6\%. Portanto, deve-se incessantemente investir no manejo de resíduos sólidos, com o objetivo de melhorar o serviço prestado à população e minimizar os danos sociais, ambientais e à saúde causados pelo manejo inadequado dos mesmos.

Palavras Chave: Coleta Seletiva, Aterro Sanitário, Lixões, Saneamento Básico

\begin{abstract}
A large amount of solid waste generated by the population leads to social, environmental and health problems, so it is important that the Brazilian municipalities have programs for solid waste management in order to reduce the effects of the mismanagement of these materials. Thus, the aim of this study was to characterize the solid waste management in southern Brazil. For data analysis, was used descriptive statistics. The data were obtained from IBGE, referring to the National Survey of Sanitation - year 2008. With this study, it was revealed that all the municipalities in southern Brazil have solid waste management, but there is no selective collection in $52.4 \%$ of the municipalities of Paraná State, $66.6 \%$ of the municipalities of Santa Catarina State and $66.5 \%$ the municipalities of Rio Grande do Sul State. In Brazil, the largest daily quantity of solid waste is destined for landfills, corresponding to $64.6 \%$. Therefore, it is necessary constantly invest in solid waste management, with the aim of improving the services provided to the population and minimize social, environmental and health damage caused by improper management of solid waste.
\end{abstract}

Keywords: selective collection, landfill, dumps, sanitation 


\section{INTRODUÇÃO}

O desenvolvimento das cidades e o crescimento da população provocaram aumento no consumo, especialmente por pessoas que possuem maior poder aquisitivo. Em decorrência disso, houve o crescimento da quantidade de resíduos gerados (CANTÓIA e LEAL, 2011). Corroborando, Besen (2011) afirma que o aumento dos resíduos gerados origina-se do crescimento da população, do acelerado processo de urbanização, das alterações tecnológicas e do avanço das condições socioeconômicas do mundo.

Nesse contexto, o ambiente urbano é um dos principais locais responsáveis por sofrer impactos ambientais e sociais, onde se desenvolve o sistema capitalista que possui como pressuposto o consumo de produtos sofisticados, os quais são facilmente descartados pelos consumidores, desse modo, aumentando a quantidade de resíduos nas regiões brasileiras (LIMA e COSTA, 2011).A elevada quantidade de resíduos sólidos tem ocasionado problemas de âmbito social, ambiental, econômico e administrativo, os quais estão passando pela grande dificuldade de implementar uma disposição adequada dos resíduos (VELOSO et al., 2009).

Cabe ressaltar que o problema do gerenciamento dos resíduos é uma das maiores dificuldades encontradas pela administração pública ou privada, pelo fato do lixo ser gerado por várias atividades humanas, ocasionando graves problemas políticos, sociais, técnicos, ambientais e de saúde (LOPES, 2003). Em continuidade a isso, "os resíduos, quando não recebem o tratamento adequado, podem causar prejuízos ambientais e sociais sem precedentes" (LIMA e COSTA, p.92, 2011).

As Diretrizes Nacionais para o Saneamento Básico (BRASIL, 2007) possuem como marco de referência a lei 11.445, de janeiro de 2007. A referida lei determina que para o manejo de resíduos sólidos sejam estabelecidas atividades de coleta, transporte, transbordo, tratamento e disposição final dos resíduos sólidos domésticos (CALDEIRA, REZENDE e HELLER, 2009). Nas áreas urbanas, o gerenciamento dos resíduos sólidos é realizado pela administração pública e baseia-se na coleta e disposição dos resíduos em locais adequados (LIMA, LIMA e SILVA, 2012). Entretanto, a ausência de políticas públicas direcionadas para o setor de resíduos sólidos demonstra a necessidade de um envolvimento maior da sociedade, aliado a uma integração eficaz entre os governos federal, estadual e municipal, com o intuito de diminuir os problemas decorrentes destes resíduos (LOPES, 2003).

Pode-se mencionar que alguns dos problemas de uma gestão deficiente de resíduos sólidos são a poluição atmosférica, os odores e gases nocivos, a poluição hídrica ocasionada pelo chorume de "lixões" e do lançamento dos resíduos no ambiente; a degradação e contaminação do solo, a desvalorização imobiliária das áreas próximas aos locais de disposição de resíduos e a proliferação de doenças (ANDRADE e FERREIRA, 2011). É crescente a preocupação mundial acerca do assunto de crescimento da produção de resíduos sólidos, particularmente os domiciliares, do gerenciamento inadequado e da ausência de locais destinados a disposição final destes resíduos (JACOBI e BESEN, 2011). A solução dos problemas desde a geração até a disposição final dos resíduos urbanos está vinculada à população envolvida, aos hábitos, às condições econômicas, à disponibilidade de locais e ao acesso a tecnologias de tratamento e disposição final dos resíduos sólidos (PAVAN, 2010).

Nesse enfoque, é possível controlar e diminuir os impactos gerados por meio de soluções acertadas de tratamento e disposição de resíduos com a implantação da gestão de resíduos sólidos nos municípios (MAGALHÃES, FILHO e PEREIRA, 2011). Os mesmos autores ressaltam que para se implantar ações destinadas ao gerenciamento dos resíduos é necessário estabelecer metas e objetivos destinados a buscar melhorias contínuas por meio da sensibilização da população e da adoção de tecnologias com o intuito de diminuírem os impactos da gestão imprópria dos resíduos. Diante do exposto, o objetivo deste estudo é caracterizar o manejo de resíduos sólidos na região Sul do Brasil.

\section{MATERIAL E MÉTODOS}

A pesquisa caracteriza-se como quantitativa, descritiva, com dados provenientes do site do Instituto Brasileiro de Geografia e Estatística referentes à Pesquisa Nacional de Saneamento Básico de 2008 (IBGE, 2013). Foram analisados dados sobre manejo de resíduos sólidos de todos os municípios dos estados da região sul do Brasil (Rio Grande do Sul, Santa Catarina e Paraná), bem como alguns dados de alcance nacional. Para a análise dos dados, foi utilizado o software Statistical Package for the Social Sciences (SPSS), Version 12.0, com o propósito de realizar estatística descritiva. Para Guimarães et al. (p.22, 2009), estatística descritiva compreende "os métodos que envolvem 
a coleta, a apresentação e a caracterização de um conjunto de dados, de modo a descrever apropriadamente as várias características desse conjunto".

\section{RESULTADOS E DISCUSSÃO}

De acordo com Medeiros et al. (p.4, 2009), a geração de resíduos sólidos nas áreas urbanas é "um dos principais reflexos do crescimento populacional associado às mudanças dos hábitos de consumo da população, sendo o seu principal destino a sua disposição em lixões, aterros controlados e aterros sanitários". Existem vários fatores que têm comprometido a questão do lixo, tais como, o crescimento da densidade populacional, o padrão de vida da população, o acréscimo de produção de materiais e o consequente crescimento dos resíduos gerados (SILVA e DONAIRE, 2007).

$\mathrm{Na}$ Tabela 1, verifica-se que todos os municípios do Paraná, Santa Catarina e Rio Grande do Sul possuem manejo de resíduos sólidos. No entanto, considerando a existência de coleta seletiva, observa-se que no Paraná, em Santa Catarina e no Rio Grande do Sul predomina a não existência desta coleta nos municípios de cada estado, na ordem de $52,5 \%, 66,6 \%$ e $66,5 \%$, respectivamente. No nível nacional, constata-se que $82,1 \%$ dos municípios não possui coleta seletiva.

De acordo com Siqueira e Moraes (2009), o gerenciamento dos resíduos sólidos está ligado com o processo de conscientização das pessoas em relação aos padrões de consumo, da importância da coleta seletiva e reutilização de materiais. Corroborando, Bringhenti e Günther (2011) dizem que é essencial que a comunidade seja sensibilizada, motivada e entenda os conceitos ligados a prática de coleta seletiva, para garantir a viabilidade e continuidade desse processo, para alcançar os resultados esperados e assegurar a sustentabilidade.

Percebeu-se que no Paraná $84,2 \%$ dos municípios possuem catadores na zona urbana, em Santa Catarina 58,0\% e no Rio Grande do Sul 53,8\% (Tabela 1). Dados no nível nacional apontam que $50,9 \%$ dos municípios não possuem catadores na zona urbana. O catador pode ser considerado como a pessoa que está lutando por sua sobrevivência no meio urbano, onde sua matéria-prima é disponível em grande quantidade e gratuita e várias pessoas pagam para se livrar dela (MAGERA, 2005). Conforme Andrade e Ferreira (p. 18, 2011), os catadores "andam pelas ruas revirando os sacos de lixo, mexendo nas lixeiras e expondo-se a riscos sérios à saúde".

$\mathrm{Na}$ Tabela 2, observa-se que o Paraná possui, na comparação com os demais estados da Região Sul, maior quantidade de municípios que se beneficiam com os tipos de serviços de manejo de resíduos sólidos. Sendo que em todos os 399 municípios do Paraná existe a coleta domiciliar regular de lixo (Tabela 2). Cabe ressaltar que o Rio Grande do Sul somente possui maior abrangência nos municípios segundo o tipo de coleta de resíduos sólidos especiais (de saúde e industriais) $(84,75)$; limpeza de praias $(9,9 \%)$ e tratamento de resíduos sólidos $(36,5 \%)$, comparativamente com o Paraná e Santa Catarina. Quando considerados dados para todo o país, percebe-se que apenas $16,8 \%$ dos municípios realizam tratamento de resíduos sólidos, $17,8 \%$ municípios realizam coleta seletiva de resíduos sólidos recicláveis e $17,8 \%$ de municípios realizam triagem de resíduos sólidos recicláveis.

Tabela 1 - Número de municípios com serviços de manejo de resíduos sólidos (Unidades) no Sul do Brasil -2008

\begin{tabular}{|c|c|c|c|c|}
\hline \multirow{2}{*}{ Serviço de manejo de resíduos sólidos } & $\mathrm{PR}$ & $\mathrm{SC}$ & $\mathrm{RS}$ & $\overline{\mathrm{BR}}$ \\
\hline & $\mathrm{N}(\%)$ & $\mathrm{N}(\%)$ & $\mathrm{N}(\%)$ & $\mathrm{N}(\%)$ \\
\hline \multicolumn{5}{|l|}{ Existência de manejo de resíduos } \\
\hline Existe & $399(100,0)$ & $293(100,0)$ & $496(100,0)$ & $5562(99,9)$ \\
\hline Não existe & 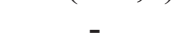 & & 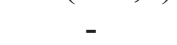 & $2(0,1)$ \\
\hline Existência de coleta seletiva & $190(47,6)$ & $98(33,4)$ & $166(33,5)$ & $994(17,9)$ \\
\hline Não existe & $209(52,4)$ & $195(66,6)$ & $330(66,5)$ & $4568(82,1)$ \\
\hline \multicolumn{5}{|l|}{$\begin{array}{l}\text { Existência de catadores na zona } \\
\text { urbana }\end{array}$} \\
\hline Existe & $336(84,2)$ & $170(58,0)$ & $267(53,8)$ & $2730(49,1)$ \\
\hline Não existe & $63(15,8)$ & $123(42,0)$ & $229(46,2)$ & $2832(50,9)$ \\
\hline Total de Municípios & 399 & 293 & 496 & $5564(100)$ \\
\hline
\end{tabular}

FONTE: Elaborada com base nos dados extraídos da Pesquisa Nacional de Saneamento Básico de 2008. 
Tabela 2 - Número de municípios com serviços de manejo de resíduos sólidos (Unidades), por natureza dos serviços na Região Sul do Brasil - 2008.

\begin{tabular}{lcccc}
\hline Natureza dos serviços de manejo de & PR & SC & RS & BR \\
\cline { 2 - 5 } resíduos sólidos* & $\mathrm{N}(\%)$ & $\mathrm{N}(\%)$ & $\mathrm{N}(\%)$ & $\mathrm{N}(\%)$ \\
\hline Coleta domiciliar regular de lixo & $399(100,0)$ & $292(99,7)$ & $494(99,6)$ & $5540(99,6)$ \\
Varrição de vias e logradouros públicos & $380(95,2)$ & $265(90,4)$ & $442(89,1)$ & $5403(97,1)$ \\
Coleta regular de r.s. das vias e logradouros & $371(93,0)$ & $265(90,4)$ & $468(94,4)$ & $5446(97,9)$ \\
públicos & $193(48,4)$ & $93(31,7)$ & $168(33,9)$ & $993(17,8)$ \\
Coleta seletiva de r.s. recicláveis & $163(40,9)$ & $77(26,3)$ & $174(35,1)$ & $990(17,8)$ \\
Triagem de r.s. recicláveis & $267(66,9)$ & $110(37,5)$ & $256(51,6)$ & $3985(71,6)$ \\
Coleta de resíduos de construção e & $333(83,5)$ & $242(82,6)$ & $420(84,7)$ & $4464(80,2)$ \\
demolição & $379(95,0)$ & $262(89,4)$ & $455(91,7)$ & $5404(97,1)$ \\
Coleta de r.s. especiais (de saúde e & $190(47,6)$ & $48(16,4)$ & $160(32,3)$ & $2306(41,4)$ \\
industriais) & $22(5,5)$ & $21(7,2)$ & $49(9,9)$ & $572(10,3)$ \\
Capina de vias e logradouros públicos & $144(36,1)$ & $30(10,2)$ & $102(20,6)$ & $3333(59,9)$ \\
Coleta de r.s. volumosos especiais & $328(82,2)$ & $204(69,6)$ & $383(77,2)$ & $4834(86,9)$ \\
Limpeza de praias & $352(88,2)$ & $239(81,6)$ & $406(81,9)$ & $5021(90,2)$ \\
Limpeza de feiras e/ou mercados públicos & $364(91,2)$ & $248(84,6)$ & $429(86,5)$ & $4091(73,5)$ \\
Remoção de animais mortos & $360(90,2)$ & $241(82,3)$ & $433(87,3)$ & $5088(91,4)$ \\
Poda de árvores & $129(32,3)$ & $54(18,4)$ & $181(36,5)$ & $936(16,8)$ \\
Limpeza de bocas-de-lobo & $350(87,7)$ & $64(21,8)$ & $247(49,8)$ & $4584(82,4)$ \\
Pintura de guias & $399(100)$ & $293(100)$ & $496(100)$ & $5564(100)$ \\
Tratamento de r.s. & & &
\end{tabular}

*Um mesmo município pode apresentar mais de um tipo de serviço; r.s. = Resíduos Sólidos

FONTE: Elaborada com base nos dados extraídos da Pesquisa Nacional de Saneamento Básico de 2008

Tabela 3 - Número de sedes de município com serviço de coleta regular de resíduos sólidos nas vias e logradouros públicos (unidades) por frequência de coleta no Sul do Brasil - 2008.

\begin{tabular}{lcccc}
\hline \multicolumn{1}{c}{ Frequência de coleta } & $\mathrm{PR}$ & $\mathrm{SC}$ & $\mathrm{RS}$ & $\mathrm{NR}$ \\
\hline Coleta no centro & & $\mathrm{N}(\%)$ & & $\mathrm{N}(\%)$ \\
$\quad$ Diária & $160(44,7)$ & $93(37,5)$ & $155(34,4)$ & $3195(60,4)$ \\
$\quad$ Três vezes por semana & $80(22,3)$ & $72(29,0)$ & $131(29,1)$ & $962(18,2)$ \\
$\quad$ Duas vezes por semana & $40(11,2)$ & $37(14,9)$ & $73(16,2)$ & $498(9,4)$ \\
$\quad$ Uma vez por semana & $52(14,5)$ & $34(13,7)$ & $88(19,6)$ & $478(9,0)$ \\
$\quad$ Outra & $33(9,2)$ & $31(12,5)$ & $65(14,4)$ & $267(5,0)$ \\
\hline Total de sedes de municípios no centro & $358(100)$ & $248(100)$ & $450(100)$ & $5291(100)$ \\
\hline Coleta nos bairros & & & & \\
$\quad$ Diária & $59(18,4)$ & $21(10,0)$ & $67(17,0)$ & $1919(39,5)$ \\
$\quad$ Três vezes por semana & $110(34,4)$ & $94(45,0)$ & $166(42,1)$ & $1440(29,7)$ \\
$\quad$ Duas vezes por semana & $57(17,8)$ & $40(19,1)$ & $80(20,3)$ & $720(14,8)$ \\
Uma vez por semana & $66(20,6)$ & $31(14,8)$ & $70(17,8)$ & $576(11,9)$ \\
$\quad$ Outra & $36(11,3)$ & $36(17,2)$ & $63(16,0)$ & $325(6,7)$ \\
$\quad$ Não faz coleta & $38(11,9)$ & $39(18,7)$ & $56(14,2)$ & $435(9,0)$ \\
\hline Total de sedes de município nos bairros & $320(100)$ & $209(100)$ & $394(100)$ & $4856(100)$ \\
\hline
\end{tabular}

FONTE: Elaborada com base nos dados extraídos da Pesquisa Nacional de Saneamento Básico de 2008 
Tabela 4 - Número de município com serviço de manejo de resíduos sólidos (unidades por cem mil habitantes) por forma de execução do serviço no Sul do Brasil - 2008.

\begin{tabular}{lcccc}
\hline \multicolumn{1}{c}{ Forma de execução do serviço } & $\mathrm{PR}$ & $\mathrm{SC}$ & $\mathrm{RS}$ & $\mathrm{BR}$ \\
\cline { 2 - 5 } Prefeitura e outras entidades são as executoras & $\mathrm{N}(\%)$ & $\mathrm{N}(\%)$ & $\mathrm{N}(\%)$ & $\mathrm{N}(\%)$ \\
do serviço & $286(71,7)$ & $199(67,9)$ & $392(79,0)$ & $3285(59,0)$ \\
Outras entidades são as executoras do serviço & $43(10,8)$ & $58(19,8)$ & $63(12,7)$ & $542(9,7)$ \\
Prefeitura é a única executora & $70(17,5)$ & $36(12,3)$ & $41(8,3)$ & $1735(31,2)$ \\
\hline Total de municípios & $399(100)$ & $293(100)$ & $496(100)$ & $5564(100)$ \\
\hline
\end{tabular}

Fonte: Elaborada com base nos dados extraídos da Pesquisa Nacional de Saneamento Básico de 2008

De acordo com a Tabela 3, percebe-se que a coleta diária de resíduos sólidos nas vias e logradouros públicos no centro é mais frequente do que a coleta diária nos bairros nos municípios dos estados da Região Sul. Andrade e Ferreira (2011) afirmam que nos municípios a coleta de resíduos sólidos é realizada com eficiência nos bairros nobres ou nas ruas principais, enquanto que, nas áreas menos privilegiadas, a coleta de resíduos sólidos é irregular ou ineficiente.

Adicionando-se a isso, no centro predominou a coleta diária, a qual ocorre em $44,7 \%$ das sedes de municípios no Paraná, 37,5\% em Santa Catarina, 34,4\% no Rio Grande do Sul e 60,4\% das sedes de municípios no país (Tabela 3). Em relação a coleta nos bairros, prevaleceu o maior percentual de sedes de municípios que possuem coleta de resíduos sólidos três vezes por semana no Paraná $(34,4 \%)$, em Santa Catarina $(45,0 \%)$ e no Rio Grande do Sul (42,1\%). Enquanto que, no Brasil, a coleta diária de bairros predominou, na ordem de $39,5 \%$, seguido pela coleta três vezes por semana, correspondendo a $29,7 \%$.

No Brasil, os responsáveis pela administração dos serviços de limpeza urbana são os municípios, os quais possuem a opção de gerenciar este aspecto por meio de uma empresa pública ou privada e de forma consorciada com outros municípios (SILVA e DONAIRE, 2007). Diante disso, quando considerado o aspecto da forma de execução do serviço de manejo de resíduos sólidos (Tabela 4), prevaleceram nos três estados da região e no nível nacional a prefeitura e outras entidades como executoras do serviço de manejo de resíduos sólidos, sendo no do Paraná 71,7\%, em Santa Catarina 67,9\%, no Rio Grande do Sul

Tabela 5 - Número de entidades prestadoras de serviços de manejo de resíduos sólidos no Sul do Brasil - 2008

\begin{tabular}{lcccc}
\hline Serviço de manejo de resíduos sólidos & $\mathrm{NR}$ & $\mathrm{SC}$ & $\mathrm{RS}$ & $\mathrm{NR}$ \\
\hline Tipo de natureza jurídica da entidade & & & & $\mathrm{N}(\%)$ \\
Empresa privada & $437(51,7)$ & $334(55,3)$ & $703(60,2)$ & $2830(34,5)$ \\
Administração direta do poder público & $364(43,0)$ & $252(41,7)$ & $440(37,7)$ & $5027(61,2)$ \\
Autarquia & $1(0,1)$ & $4(0,7)$ & $2(0,2)$ & $44(0,5)$ \\
Associação & $28(3,3)$ & $5(0,8)$ & $5(0,4)$ & $112(1,4)$ \\
Empresa pública & $7(0,8)$ & $4(0,7)$ & $5(0,4)$ & $72(0,9)$ \\
Sociedade de economia mista & $5(0,6)$ & $1(0,2)$ & $2(0,2)$ & $23(0,3)$ \\
Consórcio público & $1(0,1)$ & $2(0,3)$ & $3(0,3)$ & $46(0,6)$ \\
Fundação & $1(0,1)$ & - & - & $10(0,1)$ \\
Outra & $2(0,2)$ & $2(0,3)$ & $7(0,6)$ & $44(0,5)$ \\
Esfera administrativa & & & & \\
Privada & $467(55,2)$ & $340(56,3)$ & $715(61,3)$ & $2989(36,4)$ \\
Municipal & $375(44,3)$ & $258(42,7)$ & $446(38,2)$ & $5135(62,6)$ \\
Estadual & $3(0,4)$ & $4(0,7)$ & $3(0,3)$ & $34(0,4)$ \\
Intermunicipal & $1(0,1)$ & $2(0,3)$ & $3(0,3)$ & $47(0,6)$ \\
Federal & - & - & - & $3(0,0)$ \\
\hline Total & $846(100,0)$ & $604(100,0)$ & $1.167(100,0)$ & $8208(100)$ \\
\hline
\end{tabular}

Fonte: Elaborada com base nos dados extraídos da Pesquisa Nacional de Saneamento Básico de 2008 
$79,0 \%$, e no Brasil como um todo $59,0 \%$.

As prefeituras e a coletividade possuem o dever de destinar corretamente os resíduos sólidos, gerando boas condições de saúde pública e de qualidade ambiental, entretanto, uma parcela significativa da população não é atendida pelos referidos serviços (CALDEIRA, REZENDE e HELLER, 2009).

Em relação à natureza jurídica da entidade prestadora de serviço de manejo de resíduos sólidos nos municípios dos estados da região Sul, percebeu-se que o maior percentual de municípios possui uma empresa privada como executora deste serviço, na ordem de 51,7\% no Paraná, 55,3\% em Santa Catarina e 60,2\% no Rio Grande do Sul. Entretanto, no Brasil houve a predominância de entidades ligadas a administração direta do poder público, na ordem de $61,2 \%$. Em continuidade a isso, a esfera administrativa prestadora de serviços de manejo sólido que prevaleceu nos municípios foi a privada, correspondendo a 55,2\% no Paraná,
$56,3 \%$ em Santa Catarina e 61,3\% no Rio Grande do Sul. No nível nacional, prevaleceu a esfera municipal (62,6\%) (Tabela 5). Para Andrade e Ferreira (2011) existe uma crescente participação de empresas privadas na execução das atividades relacionadas à limpeza urbana por meio de terceirização e concessão nos municípios de médio e grande porte, proporcionando melhorias na prestação dos serviços e aumento dos custos nesse processo.

Quando observada a Tabela 6, onde estão apresentadas as unidades de destino dos resíduos sólidos domiciliares e/ou públicos segundo os grupos de tamanho dos municípios e a densidade populacional, verifica-se que naqueles com menos de 100.000 habitantes e densidade menor que 80 hab. $/ \mathrm{km}^{2}$ bem como nos que têm mais de $50.000 \mathrm{a}$ 100.000 habitantes e densidade menor que 80 hab./ $\mathrm{km}^{2}$, o destino final é o "lixão", com percentual entre 41 a $45,5 \%$ dos municípios. Concentram-se na categoria "aterro sanitário" os municípios com

Tabela 6 - Municípios com serviço de manejo de resíduos sólidos por unidade de destino dos resíduos sólidos domiciliares e/ou públicos segundo os grupos de tamanho dos municípios e a densidade populacional - Brasil - 2008.

\begin{tabular}{|c|c|c|c|c|c|c|c|c|c|c|}
\hline $\begin{array}{l}\text { Unidade de destino dos resíduos } \\
\text { sólidos domiciliares e/ou públicos }\end{array}$ & & LI & VA & $\mathrm{AC}$ & AS & $\mathrm{UC}$ & UTRR & UTI & $\mathrm{O}$ & Total \\
\hline $\begin{array}{l}\text { Até } 50.000 \text { hab. e densidade menor } \\
\text { que } 80 \text { hab. } / \mathrm{km}^{2}\end{array}$ & $\begin{array}{l}\mathrm{N} \\
(\%)\end{array}$ & $\begin{array}{c}2402 \\
(45,5)\end{array}$ & $\begin{array}{c}11 \\
(0,2)\end{array}$ & $\begin{array}{c}1005 \\
(19,0)\end{array}$ & $\begin{array}{l}1098 \\
(20,8)\end{array}$ & $\begin{array}{l}166 \\
(3,1)\end{array}$ & $\begin{array}{l}470 \\
(8,9)\end{array}$ & $\begin{array}{c}18 \\
(0,3)\end{array}$ & $\begin{array}{c}111 \\
(2,1)\end{array}$ & $\begin{array}{c}5281 \\
(100,0)\end{array}$ \\
\hline $\begin{array}{l}\text { Até } 50.000 \text { hab. e densidade maior } \\
\text { que } 80 \text { hab. } / \mathrm{km}^{2}\end{array}$ & $\begin{array}{l}\mathrm{N} \\
(\%)\end{array}$ & $\begin{array}{c}241 \\
(41,3)\end{array}$ & $(-)$ & $\begin{array}{c}91 \\
(15,6)\end{array}$ & $\begin{array}{c}159 \\
(27,3)\end{array}$ & $\begin{array}{c}15 \\
(2,6)\end{array}$ & $\begin{array}{c}64 \\
(11,0)\end{array}$ & $\begin{array}{c}5 \\
(0,9)\end{array}$ & $\begin{array}{c}8 \\
(1,4)\end{array}$ & $\begin{array}{c}583 \\
(100,0)\end{array}$ \\
\hline $\begin{array}{l}\text { Mais de } 50.000 \text { a } 100.000 \text { hab. e } \\
\text { densidade menor que } 80 \text { hab. } / \mathrm{km}^{2}\end{array}$ & $\begin{array}{l}\mathrm{N} \\
(\%)\end{array}$ & $\begin{array}{c}84 \\
(42,4)\end{array}$ & $\begin{array}{c}2 \\
(1,0)\end{array}$ & $\begin{array}{c}43 \\
(21,7)\end{array}$ & $\begin{array}{c}39 \\
(19,7)\end{array}$ & $\begin{array}{c}4 \\
(2,0)\end{array}$ & $\begin{array}{c}21 \\
(10,6)\end{array}$ & $\begin{array}{c}1 \\
(0,5)\end{array}$ & $\begin{array}{c}4 \\
(2,0)\end{array}$ & $\begin{array}{l}198 \\
(100)\end{array}$ \\
\hline $\begin{array}{l}\text { Mais de } 50.000 \text { a } 100.000 \text { hab. e } \\
\text { densidade maior que } 80 \text { hab. } / \mathrm{km}^{2}\end{array}$ & $\begin{array}{l}\mathrm{N} \\
(\%)\end{array}$ & $\begin{array}{c}41 \\
(19,1)\end{array}$ & $(-)$ & $\begin{array}{c}41 \\
(19,1)\end{array}$ & $\begin{array}{c}92 \\
(42,8)\end{array}$ & $\begin{array}{c}5 \\
(2,3)\end{array}$ & $\begin{array}{c}29 \\
(13,5)\end{array}$ & $\begin{array}{c}3 \\
(1,4)\end{array}$ & $\begin{array}{c}4 \\
(1,9)\end{array}$ & $\begin{array}{c}215 \\
(100,0)\end{array}$ \\
\hline $\begin{array}{l}\text { Mais de } 100.000 \text { a } 300.000 \text { hab. e } \\
\text { densidade menor que } 80 \text { hab. } / \mathrm{km}^{2}\end{array}$ & $\begin{array}{c}\mathrm{N} \\
(\%)\end{array}$ & $\begin{array}{c}19 \\
(37,3)\end{array}$ & $\begin{array}{l}- \\
(-)\end{array}$ & $\begin{array}{c}11 \\
(21,6)\end{array}$ & $\begin{array}{c}14 \\
(27,5)\end{array}$ & $\begin{array}{c}1 \\
(2,0)\end{array}$ & $\begin{array}{c}5 \\
(9,8)\end{array}$ & $\begin{array}{c}1 \\
(2,0)\end{array}$ & $(-)$ & $\begin{array}{c}51 \\
(100)\end{array}$ \\
\hline $\begin{array}{l}\text { Mais de } 100000 \text { a } 300000 \text { hab. e } \\
\text { densidade maior que } 80 \text { hab. } / \mathrm{km}^{2}\end{array}$ & $\begin{array}{l}\mathrm{N} \\
(\%)\end{array}$ & $\begin{array}{c}15 \\
(8,3)\end{array}$ & $\begin{array}{c}1 \\
(0,6)\end{array}$ & $\begin{array}{c}35 \\
(19,3)\end{array}$ & $\begin{array}{c}85 \\
(47,0)\end{array}$ & $\begin{array}{c}10 \\
(5,5)\end{array}$ & $\begin{array}{c}29 \\
(16,0)\end{array}$ & $\begin{array}{c}2 \\
(1,1)\end{array}$ & $\begin{array}{c}4 \\
(2,2)\end{array}$ & $\begin{array}{c}181 \\
(100,0)\end{array}$ \\
\hline Mais de 300.000 a 500.000 hab. & $\begin{array}{l}\mathrm{N} \\
(\%)\end{array}$ & $\begin{array}{c}4 \\
(6,7)\end{array}$ & $(-)$ & $\begin{array}{c}16 \\
(26,7)\end{array}$ & $\begin{array}{c}24 \\
(40,0)\end{array}$ & $\begin{array}{c}4 \\
(6,7)\end{array}$ & $\begin{array}{c}9 \\
(15,0)\end{array}$ & $\begin{array}{c}2 \\
(3,3)\end{array}$ & $\begin{array}{c}1 \\
(1,7)\end{array}$ & $\begin{array}{c}60 \\
(100)\end{array}$ \\
\hline Mais de 500.000 a 1.000 .000 hab. & $\begin{array}{c}\mathrm{N} \\
(\%)\end{array}$ & $\begin{array}{c}3 \\
(8,3) \\
\end{array}$ & $(-)$ & $\begin{array}{c}7 \\
(19,4) \\
\end{array}$ & $\begin{array}{c}16 \\
(44,4) \\
\end{array}$ & $(-)$ & $\begin{array}{c}8 \\
(22,2) \\
\end{array}$ & $\begin{array}{c}1 \\
(2,8) \\
\end{array}$ & $\begin{array}{c}1 \\
(2,8) \\
\end{array}$ & $\begin{array}{c}36 \\
(100,0) \\
\end{array}$ \\
\hline Mais de 1.000 .000 hab. & $\begin{array}{l}\mathrm{N} \\
(\%)\end{array}$ & $\begin{array}{c}1 \\
(2,9)\end{array}$ & $(-)$ & $\begin{array}{c}5 \\
(14,3)\end{array}$ & $\begin{array}{c}13 \\
(37,1)\end{array}$ & $\begin{array}{c}6 \\
(17,1)\end{array}$ & $\begin{array}{c}8 \\
(22,9)\end{array}$ & $\begin{array}{c}1 \\
(2,9)\end{array}$ & $\begin{array}{c}1 \\
(2,9)\end{array}$ & $\begin{array}{c}35 \\
(100,0)\end{array}$ \\
\hline $\begin{array}{l}\text { Quantidade diária de resíduos } \\
\text { sólidos }\end{array}$ & $\begin{array}{l}\mathrm{N} \\
(\%)\end{array}$ & $\begin{array}{l}2810 \\
(42,3)\end{array}$ & $\begin{array}{c}14 \\
(0,2)\end{array}$ & $\begin{array}{l}1254 \\
(18,9)\end{array}$ & $\begin{array}{l}1540 \\
(23,2)\end{array}$ & $\begin{array}{c}211 \\
(3,2)\end{array}$ & $\begin{array}{c}643 \\
(9,7)\end{array}$ & $\begin{array}{c}34 \\
(0,5)\end{array}$ & $\begin{array}{l}134 \\
(2,0)\end{array}$ & $\begin{array}{c}6640 \\
(100,0)\end{array}$ \\
\hline
\end{tabular}

$\mathrm{LI}=\mathrm{Lixão} ; \mathrm{VA}=$ Vazadouro em áreas alagadas ou alagáveis; $\mathrm{AC}=$ Aterro controlado; AS= Aterro sanitário; UC= Unidade de compostagem de resíduos orgânicos; UTRR= Unidade de triagem de resíduos recicláveis; UTI= Unidade de tratamento por incineração; $\mathrm{O}=$ Outra; Nota: O município pode apresentar mais de um tipo de unidade de destino dos resíduos sólidos domiciliares e/ou públicos e mais de uma unidade de destino do mesmo tipo, caso haja destinação também para outro município.

Fonte: Elaborada com base nos dados extraídos da Pesquisa Nacional de Saneamento Básico de 2008 
mais de 100.000 a 300.000 habitantes e densidade maior que $80 \mathrm{hab} . / \mathrm{km}^{2}$ e os que têm mais de 300.000 habitantes.

Percebeu-se, também, que a maior parte dos municípios do Brasil destina seus resíduos sólidos domiciliares e/ou públicos para lixões, na ordem de $42,3 \%$, e em seguida o destino mais ocupado pelos municípios é o aterro sanitário, correspondendo a 23,2\%( Tabela 6). É importante salientar que o lixão é um local onde os resíduos sólidos são simplesmente lançados, sem nenhum tipo de controle sobre os resíduos ou afluentes e o aterro sanitário é um destino de resíduos sólidos que possui controle técnico e operacional constante, de maneira a não causar prejuízo à saúde pública ou ao meio ambiente (MEDEIROS et al., 2009).

Considerando a quantidade diária de resíduos sólidos, domiciliares e/ou públicos, coletados e/ou recebidos no Brasil, notou-se que em municípios com população entre 50.000 e 100.000 habitantes e densidade menor que $80 \mathrm{hab} . / \mathrm{km}^{2}$ existe maior quantidade de resíduos sólidos destinados ao lixão, enquanto que o destino de aterro sanitário possui maior quantidade de resíduos sólidos nos municípios com população entre 50.000 e 100.000 habitantes e densidade maior que 80 hab. $/ \mathrm{km}^{2}$. Além disso, a maior quantidade diária de resíduos sólidos no Brasil é destinada a aterros sanitários, na ordem de 64,6\%(Tabela 7). Andrade e Ferreira (2011) relatam que a produção de resí-

Tabela 7 - Quantidade diária de resíduos sólidos, domiciliares e/ou públicos, coletados e/ou recebidos, por unidade de destino final dos resíduos sólidos coletados e/ou recebidos, segundo os grupos de tamanho dos municípios e a densidade populacional - Brasil - 2008.

\begin{tabular}{|c|c|c|c|c|c|c|c|c|c|c|}
\hline \multicolumn{2}{|c|}{$\begin{array}{l}\text { Unidade de destino dos resíduos } \\
\text { sólidos domiciliares e/ou públicos }\end{array}$} & \multirow{2}{*}{$\begin{array}{c}\text { LI } \\
20418 \\
(46,1)\end{array}$} & \multirow{2}{*}{$\begin{array}{c}\text { VA } \\
39 \\
(0,1)\end{array}$} & \multirow{2}{*}{$\begin{array}{c}\mathrm{AC} \\
8734 \\
(19,7)\end{array}$} & \multirow{2}{*}{$\begin{array}{c}\text { AS } \\
13378 \\
(30,2)\end{array}$} & \multirow{2}{*}{$\begin{array}{c}\mathrm{UC} \\
373 \\
(0,8)\end{array}$} & \multirow{2}{*}{$\begin{array}{c}\text { UTRR } \\
897 \\
(2,0)\end{array}$} & \multirow{2}{*}{$\begin{array}{c}\text { UTI } \\
24 \\
(0,1)\end{array}$} & \multirow{2}{*}{$\begin{array}{c}\mathrm{O} \\
415 \\
(0,9)\end{array}$} & \multirow{2}{*}{$\begin{array}{c}\text { Total } \\
44278 \\
(100,0)\end{array}$} \\
\hline $\begin{array}{l}\text { Até } 50.000 \mathrm{hab} \text {. e densidade } \\
\text { menor que } 80 \mathrm{hab} . / \mathrm{km}^{2}\end{array}$ & $\begin{array}{l}\mathrm{N} \\
(\%)\end{array}$ & & & & & & & & & \\
\hline $\begin{array}{l}\text { Até } 50.000 \text { hab. e densidade } \\
\text { maior que } 80 \mathrm{hab} . / \mathrm{km}^{2}\end{array}$ & $\begin{array}{l}\mathrm{N} \\
(\%)\end{array}$ & $\begin{array}{l}11829 \\
(55,1)\end{array}$ & $\begin{array}{l}- \\
(-)\end{array}$ & $\begin{array}{l}1620 \\
(7,5)\end{array}$ & $\begin{array}{l}7617 \\
(35,5)\end{array}$ & $\begin{array}{c}95 \\
(0,4)\end{array}$ & $\begin{array}{l}282 \\
(1,3)\end{array}$ & $\begin{array}{c}5 \\
(0,0)\end{array}$ & $\begin{array}{c}36 \\
(0,2)\end{array}$ & $\begin{array}{r}21484 \\
(100,0)\end{array}$ \\
\hline $\begin{array}{l}\text { Mais de } 50.000 \text { a } 100.000 \\
\text { hab. e densidade menor que } \\
80 \mathrm{hab} . / \mathrm{km}^{2}\end{array}$ & $\begin{array}{c}\mathrm{N} \\
(\%)\end{array}$ & $\begin{array}{c}3907 \\
(48,1)\end{array}$ & $\begin{array}{c}4 \\
(0,0)\end{array}$ & $\begin{array}{l}1914 \\
(23,6)\end{array}$ & $\begin{array}{l}2040 \\
(25,1)\end{array}$ & $\begin{array}{c}34 \\
(0,4)\end{array}$ & $\begin{array}{l}174 \\
(2,1)\end{array}$ & $\begin{array}{l}10 \\
(0,1)\end{array}$ & $\begin{array}{l}33 \\
(0,4)\end{array}$ & $\begin{array}{c}8116 \\
(100,0)\end{array}$ \\
\hline $\begin{array}{l}\text { Mais de } 50.000 \text { a } 100.000 \\
\text { hab. e densidade maior que } \\
80 \text { hab. } / \mathrm{km}^{2}\end{array}$ & $\begin{array}{c}\mathrm{N} \\
(\%)\end{array}$ & $\begin{array}{c}4357 \\
(18,9)\end{array}$ & $\begin{array}{c}- \\
(-)\end{array}$ & $\begin{array}{c}2337 \\
(10,1)\end{array}$ & $\begin{array}{l}16038 \\
(69,5)\end{array}$ & $\begin{array}{c}81 \\
(0,4)\end{array}$ & $\begin{array}{l}245 \\
(1,1)\end{array}$ & $\begin{array}{l}- \\
(-)\end{array}$ & $\begin{array}{c}7 \\
(0,0)\end{array}$ & $\begin{array}{r}23065 \\
(100,0)\end{array}$ \\
\hline $\begin{array}{l}\text { Mais de } 100.000 \text { a } 300.000 \\
\text { hab. e densidade menor que } \\
80 \mathrm{hab} / \mathrm{km}^{2}\end{array}$ & $\begin{array}{l}\mathrm{N} \\
(\%)\end{array}$ & $\begin{array}{l}1566 \\
(30,8)\end{array}$ & $(-)$ & $\begin{array}{l}1266 \\
(24,9)\end{array}$ & $\begin{array}{c}2077 \\
(40,8)\end{array}$ & $\begin{array}{c}3 \\
(0,1)\end{array}$ & $\begin{array}{c}172 \\
(3,4)\end{array}$ & $\begin{array}{c}2 \\
(0,0)\end{array}$ & $(-)$ & $\begin{array}{c}5086 \\
(100,0)\end{array}$ \\
\hline $\begin{array}{l}\text { Mais de } 100.000 \text { a } 300.000 \\
\text { hab. e densidade maior que } \\
80 \text { hab. } / \mathrm{km}^{2}\end{array}$ & $\begin{array}{l}\mathrm{N} \\
(\%)\end{array}$ & $\begin{array}{l}1641 \\
(3,2)\end{array}$ & $\begin{array}{c}3 \\
(0,0)\end{array}$ & $\begin{array}{l}5620 \\
(11,0)\end{array}$ & $\begin{array}{l}42774 \\
(84,1)\end{array}$ & $\begin{array}{l}265 \\
(0,5)\end{array}$ & $\begin{array}{l}520 \\
(1,0)\end{array}$ & $\begin{array}{c}1 \\
(0,0)\end{array}$ & $\begin{array}{l}36 \\
(0,1)\end{array}$ & $\begin{array}{r}50860 \\
(100,0)\end{array}$ \\
\hline $\begin{array}{l}\text { Mais de } 300.000 \text { a } 500.000 \\
\text { hab. }\end{array}$ & $\begin{array}{l}\mathrm{N} \\
(\%)\end{array}$ & $\begin{array}{l}780 \\
(2,2)\end{array}$ & $\begin{array}{l}- \\
(-)\end{array}$ & $\begin{array}{r}6667 \\
(18,7)\end{array}$ & $\begin{array}{l}27754 \\
(77,9)\end{array}$ & $\begin{array}{l}253 \\
(0,7)\end{array}$ & $\begin{array}{l}135 \\
(0,4)\end{array}$ & $\begin{array}{c}5 \\
(0,0)\end{array}$ & $\begin{array}{l}15 \\
(0,0)\end{array}$ & $\begin{array}{r}35609 \\
(100,0)\end{array}$ \\
\hline $\begin{array}{l}\text { Mais de } 500.000 \text { a } 1.000 .000 \\
\text { hab. }\end{array}$ & $\begin{array}{l}\mathrm{N} \\
(\%)\end{array}$ & $\begin{array}{l}1200 \\
(5,6)\end{array}$ & $\begin{array}{l}- \\
(-)\end{array}$ & $\begin{array}{l}4636 \\
(21,5)\end{array}$ & $\begin{array}{l}15443 \\
(71,6)\end{array}$ & $\begin{array}{l}- \\
(-)\end{array}$ & $\begin{array}{c}291 \\
(1,3)\end{array}$ & $\begin{array}{c}3 \\
(0,0)\end{array}$ & $\begin{array}{c}4 \\
(0,0)\end{array}$ & $\begin{array}{r}21577 \\
(100,0)\end{array}$ \\
\hline Mais de 1.000 .000 hab. & $\begin{array}{c}\mathrm{N} \\
(\%)\end{array}$ & $\begin{array}{c}12 \\
(0,0)\end{array}$ & $\begin{array}{l}- \\
(-)\end{array}$ & $\begin{array}{c}7901 \\
(16,0)\end{array}$ & $\begin{array}{l}40515 \\
(81,9)\end{array}$ & $\begin{array}{c}531 \\
(1,1)\end{array}$ & $\begin{array}{l}406 \\
(0,8)\end{array}$ & $\begin{array}{l}17 \\
(0,0)\end{array}$ & $\begin{array}{l}90 \\
(0,2)\end{array}$ & $\begin{array}{c}49472 \\
(100,0)\end{array}$ \\
\hline $\begin{array}{l}\text { Quantidade diária de resíduos } \\
\text { sólidos }\end{array}$ & $\begin{array}{l}\mathrm{N} \\
(\%)\end{array}$ & $\begin{array}{l}45710 \\
(17,6)\end{array}$ & $\begin{array}{r}46 \\
(0,0)\end{array}$ & $\begin{array}{l}40695 \\
(15,7)\end{array}$ & $\begin{array}{r}167636 \\
(64,6)\end{array}$ & $\begin{array}{l}1635 \\
(0,6)\end{array}$ & $\begin{array}{l}3122 \\
(1,2)\end{array}$ & $\begin{array}{r}67 \\
(0,0)\end{array}$ & $\begin{array}{r}636 \\
(0,2)\end{array}$ & $\begin{array}{c}259547 \\
(100,)\end{array}$ \\
\hline
\end{tabular}

LI= Lixão; VA= Vazadouro em áreas alagadas ou alagáveis; $\mathrm{AC}=$ Aterro controlado; $\mathrm{AS}=$ Aterro sanitário; UC= Unidade de compostagem de resíduos orgânicos; UTRR= Unidade de triagem de resíduos recicláveis; UTI= Unidade de tratamento por incineração; $\mathrm{O}=$ Outra; Nota: A quantidade diária dos resíduos sólidos domiciliares e/ou públicos pode ser proveniente do próprio ou de outro município.

Fonte: Elaborada com base nos dados extraídos da Pesquisa Nacional de Saneamento Básico de 2008 
duos sólidos no país está crescendo em termos de quantidade e a reciclagem possui pouco efeito em relação a quantidade que é gerada e, por isso, o crescimento da coleta domiciliar e disposição dos resíduos sólidos em local adequado são etapas a serem vencidas.

\section{CONCLUSÃO}

Com este estudo foi possível concluir que mesmo que todos os municípios dos estados da região Sul possuam manejo de resíduos sólidos, existe deficiência em relação a existência de coleta seletiva na maioria destes municípios, o que é considerada uma situação preocupante e que está na contramão das práticas desejáveis para garantir a destinação mais adequada para cada tipo de resíduo, bem como o reaproveitamento dos resíduos sólidos.

Vale destacar que a prática de recolhimento de resíduos sólidos nas vias e logradouros públicos com mais frequência no centro em comparação com os bairros deve ser repensada, pois toda a população deve ter acesso igualitário em termos de coleta regular de resíduos sólidos. Diante disso, as prefeituras e entidades executoras do serviço de manejo de resíduos sólidos devem rever e melhorar o serviço de manejo de resíduos sólidos que está sendo prestado à população.

Para concluir este estudo, notou-se que a maioria dos municípios utiliza os lixões como destino dos resíduos sólidos, mas que a maior quantidade de resíduos sólidos é destinada em aterros sanitários, demonstrando que são principalmente os municípios de menor porte os que ainda não possuem aterros sanitários disponíveis para destinação destes resíduos, situação essa que precisa ser regularizada. Cabe ressaltar que se deve incessantemente investir no gerenciamento do manejo de resíduos sólidos nos municípios do Brasil, com o intuito de melhorar o serviço prestado à população e minimizar os danos sociais, ambientais e a saúde causados pelo manejo inadequado destes.

\section{REFERÊNCIAS BIBLIOGRÁFICAS}

ANDRADE, R. M.; FERREIRA, J. A. A gestão de resíduos sólidos urbanos no Brasil frente às questões da globalização. REDE - Revista Eletrônica do Prodema, Fortaleza, v. 6, n.1, p. 7-22, mar. 2011. ISSN 1982-5528.
BESEN, G. R. Coleta seletiva com inclusão de catadores: construção participativa de indicadores e índices de sustentabilidade [tese de doutorado]. São Paulo: Faculdade de Saúde Pública da USP; 2011.

BRASIL. Lei 11.445, de 5 de janeiro de 2007. Diretrizes Nacionais do Saneamento Básico. Brasília: Diário Oficial da União, 2007.

BRINGHENTI, J.R.; GÜNTHER, W.M.R. Participação social em programas de coleta seletiva de resíduos sólidos urbanos. Eng. Sanit. Ambient., v.16, n.4, out/dez, 2011, p.421-430.

CALDEIRA, M. M.; REZENDE, S.; HELLER, L. Estudo dos determinantes da coleta de resíduos sólidos urbanos em Minas Gerais. Eng. Sanit. Ambient., v.14, n.3, jul/set 2009, p. 391-400.

CANTÓIA, S. F.; LEAL, A. C. Gerenciamento de resíduos sólidos urbanos nos municípios de Presidente Prudente, Ourinhos e Assis - São Paulo, Brasil. Revista Geográfica de América Central, v.2, n. 47, 2011.

GUIMARÃES, G.; GITIRANA, V.; MARQUES, M.; CAVALCANTI, M. R. A Educação estatística na educação infantil e nos anos iniciais. ZETETIKÉ - Cempem - FE - Unicamp, v. 17, n. 32 - jul/dez 2009.

IBGE. Instituto Brasileiro de Geografia e Estatísitica. Disponível em: http://www.ibge.gov.br/home/. Acesso em: 1 de maio de 2013.

JACOBI, P. R.; BESEN, G. R. Gestão de resíduos sólidos em São Paulo: desafios da sustentabilidade. Estudos avançados, 25 (71), 2011.

LIMA, G. M.; COSTA, F. R. Gerenciamento dos resíduos sólidos urbanos no município de Rafael Fernandes-rn. GEOTemas, Pau dos Ferros, Rio Grande do Norte, Brasil, v. 1, n. 1, p. 91-103, jan./ jun., 2011.

LIMA, R., LIMA, J., SILVA, T. Roteirização em arcos com um sistema de informações geográficas para transportes: aplicação em coleta de resíduos sólidos urbanos. Journal of Transport Literature, América do Norte, 6, fev. 2012. Disponível em: http://www.pesquisaemtransportes.net.br/relit/ index.php/relit/article/view/290. Acesso em: 19 Fev. 2012. 
LOPES, A. A. Estudo da Gestão e do gerenciamento integrado dos resíduos sólidos urbanos no município de São Carlos (SP). 178f. 2003. Dissertação - (Mestrado em Ciências da Engenharia Ambiental) - Universidade de São Paulo. 2003

MAGALHÃES, I. A. L.; FILHO, M. V.; PEREIRA, S. C. M.. Gerenciamento dos resíduos sólidos urbanos do município de bocaiúva-mg: diagnóstico da situação atual e suas proposições. Engenharia Ambiental - Espírito Santo do Pinhal, v. 8, n. 3, p. 207-224, jul ./set . 2011

MAGERA, M. C.. Os empresários do lixo: análise interdisciplinar das Cooperativas de reciclagem de lixo. $2^{\mathrm{a}}$ ed. Campinas, SP: Átomo, 2005.

MEDEIROS, G. A. Diagnóstico do aterro do município de poços de caldas, no estado de minas gerais, brasil. Engenharia Ambiental - Espírito Santo do Pinhal, v. 6, n. 2, p. 003-015, mai /ago 2009.

PAVAN, M. C. O. Geração de energia a partir de resíduos sólidos urbanos: avaliação e diretrizes para tecnologias potencialmente aplicáveis no Brasil, 2010, 186f. Tese de Doutorado, Programa de Pós Graduação em Energia da Universidade de São Paulo, 2010.

SILVA, R. C.; DONAIRE, D. A gestão de resíduos sólidos urbanos: um estudo de caso na prefeitura de São Paulo. Revista Administração e Diálogo, v. 9, n. 1, 2007, p. 121-143.

SIQUEIRA, M. M.; MORAES, M. S. Saúde coletiva, resíduos sólidos urbanos e os catadores de lixo. Ciênc. saúde coletiva [online]. 2009, vol.14, n.6, pp. 2115-2122. ISSN 1413-8123.

VELOSO, A. C. O. G., et al. Estudo dos procedimentos para o gerenciamento de resíduos sólidos nos municípios da Região Hidrográfica VIII do estado do Rio de Janeiro. Boletim do Observatório Ambiental Alberto Ribeiro Lamego, Campos dos Goytacazes/RJ, v.3, n.2, p. 109-123, jul./dez. 2009. 